\title{
Efficient generation of an isolated single-cycle attosecond pulse
}

\author{
Pengfei Lan, Peixiang Lu*, Wei Cao, Xinlin Wang \\ Wuhan National Laboratory for Optoelectronics and School of Optoelectronics Science and Engineering, \\ Huazhong University of Science and Technology, Wuhan 430074, P. R. China
}

(Dated:)

\begin{abstract}
A new method for efficiently generating an isolated single-cycle attosecond pulse is proposed. It is shown that the ultraviolet (UV) attosecond pulse can be utilized as a robust tool to control the dynamics of electron wave packets (EWPs). By adding a UV attosecond pulse to an infrared (IR) few-cycle pulse at a proper time, only one return of the EWP to the parent ion is selected to effectively contribute to the harmonics, then an isolated two-cycle 130-as pulse with a bandwidth of $45 \mathrm{eV}$ is obtained. After complementing the chirp, an isolated single-cycle attosecond pulse with a duration less than 100 as seems achievable. In addition, the contribution of the quantum trajectories can be selected by adjusting the delay between the IR and UV fields. Using this method, the harmonic and attosecond pulse yields are efficiently enhanced in contrast to the scheme [G. Sansone et al., Science 314, 443 (2006)] using a few-cycle IR pulse in combination with the polarization gating technique.
\end{abstract}

PACS numbers: 42.65.Re, 32.80.Rm, 42.65.Ky

Attosecond (as) extreme ultraviolet pulses open the way to a new regime of investigating and manipulating basic ultrafast electronic processes in atoms and molecules with an unprecedented precision [1, 2, 3, $3,4,5]$. Thus the generation of attosecond pulses has attracted spectacular interest in recent years. So far high harmonic generation (HHG) is the most promising way to produce attosecond pulses. In the breakthrough work [5], a train of attosecond pulses were produced with a multi-cycle laser pulse. However, the straightforward attosecond metrology prefers an isolated attosecond pulse [6] and then much effort has been paid to extract an isolated pulse from the attosecond pulse train.

HHG is well understood in terms of the "recollision" model [7, 8]. In detail, the electron wave packet (EWP) is first produced when the atom or molecule is ionized. When the laser field drives the EWP back to the parent ion, it interferes with the bound wavefunction, producing coherent attosecond light bursts. If only a single return is possible, an isolated attosecond pulse can be obtained. It was demonstrated that the return probability of EWP to the parent ion depends sensitively on the polarization of the driving field [9]. Using polarization gating technique [9], the EWP only returns efficiently in a short time and then an isolated attosecond pulse can be produced. An alternative method to produce isolated attosecond pulses is using a few-cycle laser pulse [1, 6]. It has been shown that the highest harmonics (so-called cutoff) are only generated at the peak of the few-cycle pulse. By filtering these harmonics, an isolated attosecond pulse can be obtained. However, the bandwidth of the harmonics in the cutoff is $\sim 10 \mathrm{eV}$, which only supports to an attosecond pulse of about 250 as 2]. Currently, an intense research is afoot to push the pulse duration to even

*lupeixiang@mail.hust.edu.cn shorter time 10, 11, 12, 13, 14]. In the resent work [13], Sansone et al. produced an isolated broadband attosecond pulse with a duration of 130 as using a few-cycle (5fs) laser pulse with a modulated polarization. However, there are some flaws for enhancing the harmonic yield in this scheme, which limits the application of this attosecond pulse. That is, the available energy of the few-cycle driving laser pulse generated by the state-of-the-art hollow fiber and chirped mirror scheme is usually less than $1 \mathrm{~mJ}$. Further, fractional energy of the few-cycle pulse is lost in the polarization gating technique.

In this work, we propose a new efficient method for the generation of isolated broadband attosecond pulses using a few-cycle IR driving laser pulse in combination with a UV attosecond controlling pulse. We first analyse the HHG in the few-cycle IR field alone. Due to the ultrashort duration, the EWP is dominantly produced near the peak of the few-cycle pulse [15]. The EWP produced at $t_{1}=3.2 T_{I R}$ (marked by $R_{1}$ in Fig. 11) is driven by the IR field at the highest amplitude, and returns to the parent ion near $4 T_{I R}$ with the energy of about $3.2 U_{p}$, where $T_{I R}$ and $U_{p}$ are the period of IR field and ponderomotive energy, respectively. In the following half cycle, the EWP is produced at $t_{2}=3.75 T_{I R}$ (marked by $R_{2}$ in Fig. 11) and returns to the parent ion at $4.5 T_{I R}$ with the energy of about $2.5 U_{p}$. Therefore more than one returns $\left(R_{1}\right.$ and $\left.R_{2}\right)$ contribute to the lower harmonics (so-called plateau), and the isolated attosecond pulse is only achievable by filtering the harmonics with a bandwidth only $0.7 U_{p}(\sim 10 \mathrm{eV})$, which prevents obtaining the broadband isolated attosecond pulse with a shorter duration. If the polarization of the few-cycle pulse is modulated, the contribution from one return can be eliminated, then a broadband attosecond pulse can be obtained [12, 13, 14]. However, the efficiency is limited as discussed in the previous paragraph. In the present work, we propose an efficient method for producing a broadband isolated attosecond pulse, this is achieved by 


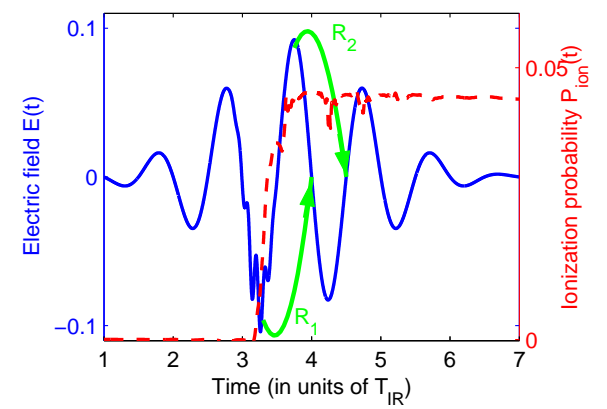

FIG. 1: (color online) Schematic illustration (solid line) of the harmonic generation process and the evolution of the ionization probability (dashed line) in the combined field of an IR few-cycle laser pulse and a UV attosecond pulse. The intensities of the IR and UV fields are $3 \times 10^{14} \mathrm{~W} / \mathrm{cm}^{2}$ and $2 \times 10^{13} \mathrm{~W} / \mathrm{cm}^{2}$, and the delay $\omega_{I R} \tau_{\text {delay }}=-1.1 \pi$.

enhancing the contribution from one return with a UV pulse. Note that the rapid advancement of HHG has made it available of a UV pulse with an intensity of about $1 \times 10^{14} \mathrm{~W} / \mathrm{cm}^{2}[16$, 17]. Such attosecond pulses provide us a robust tool to control EWPs [4], which have been used in previous works [3, 17, 18]. The schematic illustration of our method is shown in Fig. 1, An attosecond UV controlling pulse is synthesized to the few-cycle IR driving pulse at the time of $t_{1}$. Due to the high photon energy and ultrashort duration of the UV pulse, the production time and property of EWPs can be manipulated. Then, in the combined field of an IR and a UV pulses, the EWP is only effectively produced at $t_{1}$, returns to the parent ion at $t=4 T_{I R}$, and the contribution from the EWP produced at $t_{2}$ is much lower than that at $t_{1}$. Consequently, only the return $R_{1}$ is selected to effectively contribute to the harmonics, broadband harmonics and an isolated ultrashort attosecond pulse will be obtained. It should be emphasized that the few-cycle IR pulse also plays a vital role in this scheme, broadband isolated attosecond pulse can not be produced with a multi-cycle pulse [3, 18]. In contrast to the method using a single few-cycle IR pulse in combination with the polarization gating technique [13], the energy loss of the IR field is avoided in our scheme. Moreover, the UV field facilitates the ionization, then the harmonic and attosecond pulse yields are significantly enhanced.

To verify the above scheme, we perform a quantum simulation by solving the time-dependent Schrödinger equation, the Hamiltonian is $H=-\partial^{2} / 2 \partial x^{2}+V(x)-$ $x E(t)$ (in atomic units), where $E(t)$ the electric field and $V(x)$ is the atomic Coulomb potential. We choose the soft-core model within the single-active electron approximation $V(x)=-1 / \sqrt{\alpha+x^{2}}$, where $\alpha$ is a smoothing parameter, routinely employed in many studies of atomic ionization and HHG. Although our work is not intended to model a specific experiment, in order to make the discussion concrete, $\alpha$ is set to be 0.485 to retrieve the ionization energy of helium. And it has been shown that the soft-core model well describes the real atom [19], we expect our result is comparable to the HHG of helium. The electric fields of the IR and UV pulses are given by $E_{j}(t)=E_{0 j} f_{j}\left(t-\tau_{j}\right) \cos \left[\omega_{j}\left(t-\tau_{j}\right)\right], j=I R, U V . E_{0 j}$ and $\omega_{j}$ are the amplitudes and frequencies, $f(t)_{j}$ and $\tau_{j}$ are envelopes and peak positions of the pulses. Like Ref. [17], a Gaussian envelope shape is adopted and the delay of the UV pulse with respect to the IR pulse is $\tau_{\text {delay }}=\tau_{U V}-\tau_{I R}$. For the IR driving field, the intensity is $3 \times 10^{14} \mathrm{~W} / \mathrm{cm}^{2}$, the central wavelength and pulse duration are $800 \mathrm{~nm}$ and $5 \mathrm{fs}$, respectively. For the UV controlling field, the intensity is $2 \times 10^{13} \mathrm{~W} / \mathrm{cm}^{2}$, the central wavelength is $100 \mathrm{~nm}$, and pulse duration is 0.6 fs full width at half maximum, containing about 2 cycles. The time-dependent Schrödinger equation is solved with split-operator method 20]. The initial wavefunction is chosen to be the field-free ground state which is obtained by propagation in imaginary time. The ionization probability is given by $P(t)=1-\sum_{n}<\phi_{n} \mid \Psi(t)>$ where the summation runs over the bound states $\phi_{n}$. The timedependent dipole acceleration can be calculated with the Ehrenfest theorem [21], and then the harmonic spectrum is calculated by Fourier transforming the dipole acceleration.

The dashed line in Fig. 1 shows the evolution of the ionization probability in the IR driving pulse combined with an attosecond UV controlling pulse at $t_{1}=3.2 T_{I R}$. One can see that the ionization probability increases steeply at $t_{1}$ and varies slightly at other time. This indicates that the EWP is only effectively produced at $t_{1}$ which agrees with our proposal. The ionization probability is much higher than that in the IR or UV field alone, which will significantly enhance the harmonic and attosecond pulse yields. This is an effect of cross correlation of the two pulses, which can be explained as follows. The 0.6-fs UV pulse spans a bandwidth of several $\mathrm{eV}$, it can promote electronic transition from ground state to the first excited state (with the eigenenergy of -0.32). And on the other hand, IR laser photons also can assist the electronic transition. Thus, the electron in the ground state is first pumped to the excited state, and then is up-shifted and easily ionized by the IR driving field because of the low binding energy. This explanation agree well with Ref. [22]. Also, Paul et al. [23] observed significant HHG enhancement with the prepared excited atom recently. From this viewpoint, our result also can be explained that an excited atom is prepared by the UV pulse in combination with the IR pulse, and so the ionization and HHG is significantly enhanced. Note that this process is different from Ref. [3], where the photon energy of the UV pulse is higher than the ionization energy and the electron is dominantly ionized by absorbing one UV photon. Fig. 2(a) shows the harmonic spectrum by an IR few-cycle laser pulse alone (the thin line). One can see that the overall spectral structure is irregular for the harmonics bellow $52 \omega_{I R}$, and is smooth for the harmonics in the cutoff. The isolated attosecond pulse is only achievable by selecting the highest harmonics with a 


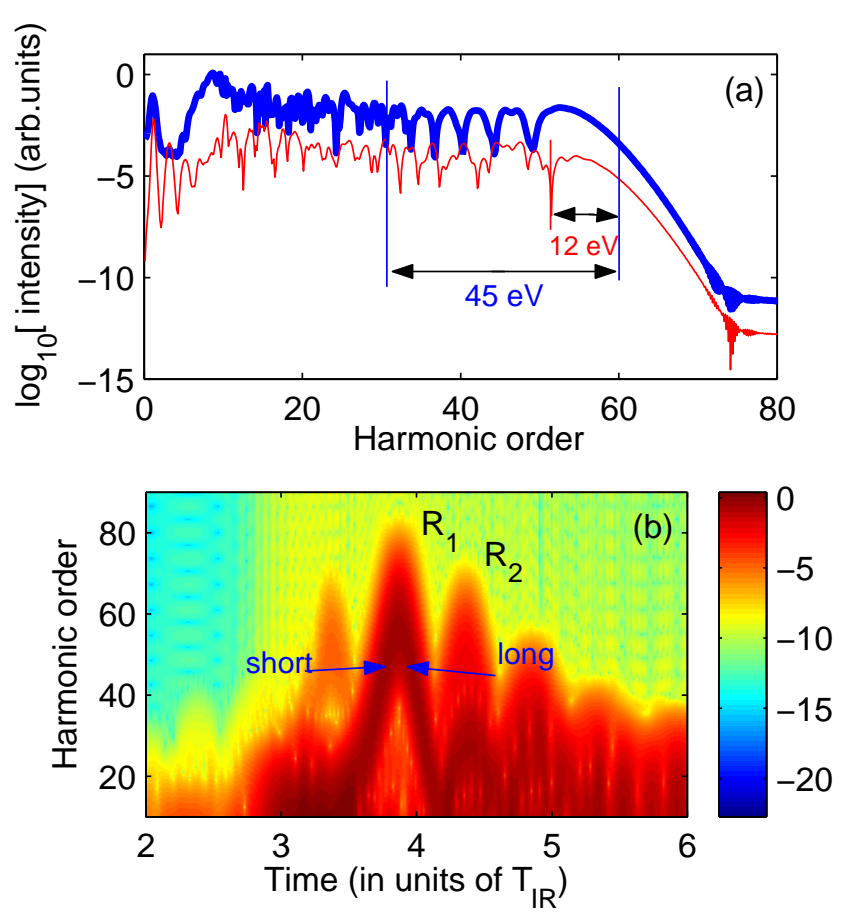

FIG. 2: (color online) (a) The harmonic spectrum by in the 5 -fs IR field alone (thin line) and in combination with a 0.6 fs UV field (bold line), respectively. (b)The time-frequency characteristics of the harmonics in the combined field. The parameters are the same with Fig. 1

bandwidth of about $12 \mathrm{eV}$. While in the combined field of an IR and UV pulses, the harmonic spectrum shows a different structure in contrast to that in the IR pulse alone. One can see that (the bold line) the harmonic spectrum is smooth and regularly modulated from $31 \omega_{I R}$. In addition, the harmonic yield is about two orders of magnitude higher than that in the IR driving field alone.

To understand the spectral structure shown in Fig. 2 (a), the time-frequency image of the harmonic spectrum [24] in the combined field is shown in Fig. 2(b). One can see that there are two dominant returns contributing to the HHG near the peak of the driving field. For the first one, the harmonics are emitted at $t=4 T_{I R}$, and the highest energy is about $60 \omega_{I R}$ which is approximately equal to $I_{p}+3.2 U_{p}$. All these features consist with the characteristics of the return $R_{1}$ and indicate that these harmonics are generated by the EWP produced at $3.2 T_{I R}$. For the second return, the harmonics are emitted at $t=4.5 T_{I R}$, which correspond to the return $R_{2}$. However, in contrast to the HHG in the IR field alone (see Fig. 1 of Ref. [15]), one can see from Fig. 2 (b) that the first return $\left(R_{1}\right)$ is highly enhanced in the combined field. Thereby only the return $R_{1}$ is selected to effectively contribute to the harmonics, and thus the harmonic spectrum becomes more regular and smooth as shown in Fig. 2(a). For the harmonics contributed from $R_{1}$, it is shown in Fig. 2(b) that there are two emission

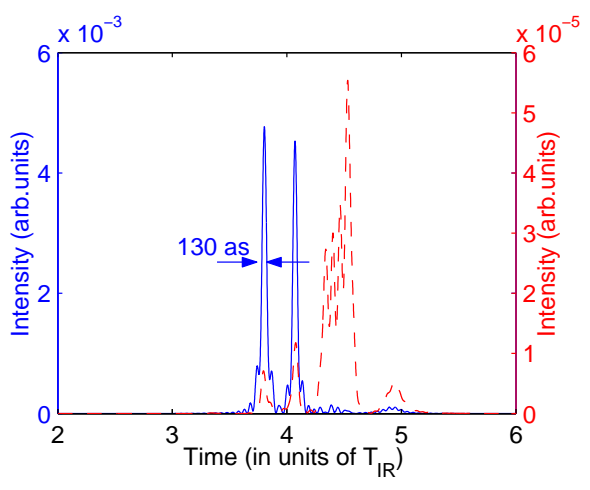

FIG. 3: (color online) Temporal profiles of the attosecond pulses by the combined field (solid line) and IR field alone (dashed line), respectively. The parameters are the same with Fig. 1

times corresponding to the same harmonic in the half optical cycle of the IR field. They correspond to the short and long quantum trajectories [7, [8]. The interference of these two quantum trajectories leads to an evident modulation in the harmonic spectrum. The spatial analogy of this phenomena is Young's two-slit experiment. This interference will be weak when eliminating one quantum trajectory, then the modulation is weak also and the harmonic spectrum will become a supercontinuum [12].

As shown in Fig. 2, the spectral structure in the combined field is different from that in the IR driving field alone, which will produce different attosecond pulses. Fig. 3 shows the temporal profiles of the attosecond pulses generated in the IR driving field alone (dashed line) and combined field (solid line) by superposing the harmonics from $31 \omega_{I R}$ to $50 \omega_{I R}$, respectively. In the IR field alone, a train of chaotic attosecond pulses are generated (see the dashed line). It is because both the return $R_{1}$ and $R_{2}$ contribute to the harmonics in the plateau, and different returns take different trajectories and return times. Hence these high harmonics are not synchronized, a train of chaotic attosecond pulses are generated and it is hard to extract an isolated attosecond pulse from this pulse train. While in the combined field, a double-peak attosecond pulse is generated near $t=4.0 T_{I R}$, which is originated from the return of $R_{1}$. In addition, the magnitude of the double-peak attosecond pulse is enhanced by about two orders in the combined field. Note that double-peak structure corresponds to the short and long trajectories [Fig. 2(b)], respectively. After selecting one trajectory, the high harmonics will be well synchronized and an isolated broadband attosecond pulse will be obtained. Generally, this can be achieved utilizing the phase-match condition. It has been demonstrated that the short trajectory can be macroscopically selected by adjusting the position of the laser focus with respect to the gas jet [13, 25]. Alternatively, the long trajectory leads to a spatially divergent radiation [26], and can be macroscopically eliminated by adding a small 


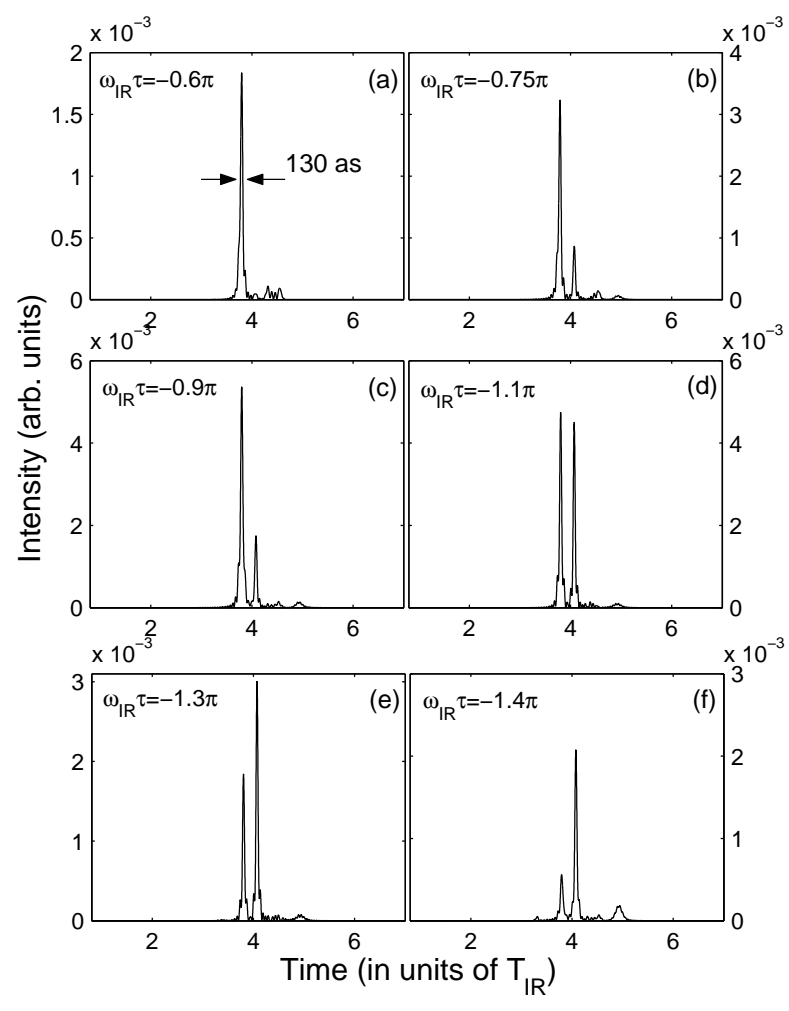

FIG. 4: Temporal profiles of the attosecond pulses generated at different delays between the IR and UV fields. An isolated broadband attosecond pulse is generated as shown in Fig. 4(a).

aperture after the harmonic generation cell [10]. With these techniques, only the short trajectory is selected and an isolated broadband attosecond pulse will be produced [11, 12, 13].

Here we show that the quantum trajectory also can be selected by adjusting the delay between the IR and UV fields, then the high harmonics can be well synchronized and an isolated broadband pulse is generated [see Fig. 4(a)]. Fig. 4 shows the temporal profiles of the attosecond pulses generated in the combined field for various delays. One can see that both the intensity and the profile of the attosecond pulses vary significantly by adjusting the delay. At $\omega_{I R} \tau_{\text {delay }}=-1.1 \pi$ [see Fig. 4(d)], both the short and long trajectories contribute to the harmonics, and two attosecond bursts with equal intensities are generated. With decreasing the delay [Fig. [4(d)-(a)], the contribution from the long trajectory becomes weak, and the intensity of the post-pulse is lower. With increasing the delay [Fig. 4(d)-(f)], the contribution from the short trajectory becomes weak, and the intensity of the pre-pulse is lower. The most fascinating result shown in Fig. 4(a) is that an isolated broadband attosecond pulse is obtained at $\omega_{I R} \tau_{\text {delay }}=-0.6 \pi$, only accompanied by a minor post-pulse with the magnitude of about $5 \%$ of the main pulse which can be ignored. The duration of this isolated attosecond pulse is only 130 as, containing only 1.95 optical cycles of the central harmonic frequency $\left(40 \omega_{I R}\right)$. In addition, it is worth emphasizing that the bandwidth of the attosecond pulse is broadened to 45 $\mathrm{eV}$. In the Fourier limit, a 90-as pulse will be obtained. However, there is an intrinsic chirp in these harmonics [see Fig. 2(b)], which prevents producing the sub-100 as pulse. This problem can be overcome by using a proper material to compensate the intrinsic chirp [10, 13]. Then an isolated single-cycle attosecond pulse with a duration less than 100 as will be obtained. The efficient generation of isolated single-cycle attosecond pulses may pave the way to investigate and control the electron dynamics in atoms and molecules with a higher precision. In addition, the few-cycle isolated attosecond pulse can serve as a tool to study the electron dynamics in atoms and molecules when processes are triggered and controlled by the electric field of the attosecond pulse rather than by the intensity profile. As in the case of few-cycle IR pulses, we expect the possibility to influence and modify the ionization mechanism of electrons upon changing the carrier-envelope phase of the attosecond pulse in the extreme UV regime.

Few-cycle Ti:sapphire laser pulses are currently available in quite a few laboratories around the world, which facilitates the implementation of our scheme. In experiments, we can split a 5-fs Ti:sapphire laser pulse to two beams. Focusing one laser beam to a gas jet (e.g. xenon), the 0.6 -fs controlling UV pulse can be produced by synthesizing the high harmonics like Refs. [1, 2], and then the generated UV pulse is combined with the other laser beam. Following, the combined field is focused to helium gas, and an isolated broadband 130-as pulse will be generated. Note that the delay between the UV pulse and IR laser pulse should be adjust (see Fig. 4). Establishing a dedicated delay shift can be done by introducing small amounts of glass in experiments.

In conclusion, a new method for the efficient generation of an isolated single-cycle attosecond pulse using a few-cycle IR driving pulse in combination with a UV attosecond controlling pulse has been proposed. It is shown that the EWP dynamics can be controlled by the UV attosecond pulse. Then only one return of the EWP to the parent ion is selected, and an isolated broadband attosecond pulse of $45 \mathrm{eV}$ is generated. In addition, the contribution of the short and long trajectories can be selected by adjusting the delay between the IR and UV fields. The results show that an isolated two-cycle attosecond pulse with a duration of 130 as is obtained. After compensating the chirp, it is expected to produce an isolated single-cycle attosecond pulse with a duration less than 100 as. For our scheme, the energy of the few-cycle IR field is efficiently used, and the UV field facilitates the ionization, thus the harmonic and attosecond pulse yields are significantly enhanced.

This work was supported by the National Natural Science Foundation of China under grant No. 10574050, the Specialized Research Fund for the Doctoral Pro- 
gram of Higher Education of China under Grant No. 20040487023 and the National Key Basic Research Spe- cial Foundation under grant No. 2006CB806006.

153901 (2003).

[16] H. Mashiko, A. Suda, K. Midorikawa, Opt. Lett. 29, 1927 (2004).

[17] A. D. Bandrauk, S. Chelkowski, N. H. Shon, Phys. Rev. Lett. 89, 283903 (2002).

[18] A. D. Bandrauk and N. H. Shon, Phys. Rev. A 66, 031401(R) (2002).

[19] Q. Su, J. H. Eberly, Phys. Rev. A 44, 5997 (1991); S. C. Rae, X. Chen, K. Burnett, Phys. Rev. A 50, 1946 (1994).

[20] M. D. Feit, J. A. Fleck, Jr, and A. Steiger, J. Comput. Phys. 47, 412 (1982).

[21] K. Burnett, V. C. Reed, J. Cooper, and P. L. Knight, Phys. Rev. A 45, 3347 (1992).

[22] K. Ishikawa, Phys. Rev. Lett. 91, 043002 (2003).

[23] P. M. Paul, et al., Phys. Rev. Lett. 94, 113906 (2005).

[24] P. Antoine, B. Piraux and A. Maquet, Phys. Rev. A 51, R1750 (1995).

[25] P. Antoine, A. L'Huillier, and M. Lewenstein, Phys. Rev. Lett. 77, 1234 (1996).

[26] M. Bellini et al., Phys. Rev. Lett. 81, 297 (1998).

[14] I. J. Sola et al., Nature Physics 2, 319 (2006).

[15] V. S. Yakovlev, and A. Scrinzi, Phys. Rev. Lett. 91, 